\title{
Patterns of corticosteroid medication use: non-adherence can be effective in milder asthma
}

\author{
Colin J. Greaves ${ }^{a, *}$, Michael E. Hyland ${ }^{b}$, David M.G. Halpin ${ }^{c}$, \\ Susan Blake ${ }^{d}$, David Seamark ${ }^{d}$
}

\author{
a Peninsula Medical School (Primary Care), Postgraduate Medical Centre, Barrack Road, \\ Exeter EX2 5DW, UK \\ b Department of Psychology, University of Plymouth, Plymouth PL4 8AA, UK \\ c Department of Respiratory Medicine, Royal Devon and Exeter Hospital (Wonford), Barrack Lane, \\ Exeter EX2 5DW, UK \\ ${ }^{\mathrm{d}}$ Honiton Group Practice, Marlpits Lane, Honiton EX14 2NY, UK \\ Received 7 June 2004; accepted 17 September 2004
}

KEYWORDS

Asthma;

Adherence;

Corticosteroid;

Medication;

Self-Care

\section{Sumirary}

Aims: To identify spectflodatierns of corticosteroid use and examine their relationsinir with astrina outcomes.

Methods: An adherence questionnaire was developed and applied in a populationbased observational survey; this compared unscheduled care visits and asthma quality of life for adherent and non-adherent patient groups within 176 patients from a semi-rural UK practice.

Results: Three main patterns of medication use were identified: Regular; Regularbut-less (Low-Dosing); and Symptom-Directed variation. For mild-to-moderate asthma (BTS treatment step 2), non-adherence produced acceptable outcomes, not significantly different from outcomes for adherent patients. For more severe asthma, regular adherence was more effective, resulting in significantly less unscheduled visits.

Conclusions: The results suggest that flexible 'symptom-directed' medication use and patient-initiated dose reduction may be viable alternatives to regular medication for a number of lower severity patients. For milder asthma, clinicians should perhaps focus their efforts on patients with poor asthma outcomes, rather than poor adherence.

(c) 2004 General Practice Airways Group. Published by Elsevier Ltd. All rights reserved.
* Corresponding author. Tel.: +44 1392 403000/798989 5165; fax: +441392 432223.

E-mail address: Colin.Greaves@pms.ac.uk (C.J. Greaves).

\section{Background}

Asthma guidelines recommend that, where patients require treatment with inhaled corticosteroids 
(ICS), these should be taken regularly every day [1], and that poor asthma outcomes are associated with non-adherence to this advice [2-4].

However, population-based studies indicate that a large number of non-adherent patients still achieve good asthma outcomes [5-7]. This implies that in some circumstances, non-adherence may be acceptable, and that some non-adherent strategies may possibly be more effective than others. As nonadherence is common in asthma [8], determining the circumstances in which non-adherence can be successful is an important question for both clinicians and patients.

Studies using dose-recording electronic inhaler devices have identified a number of common patterns of ICS medication use including regular use, apparently haphazard compliance and the taking of 'drug holidays' $[9,10]$. Reviews [11] and qualitative studies [12-14] have identified further 'strategic' patterns, which identify the patient's declared strategy for using ICS medication (e.g. minimisation of steroid use, reacting to symptoms), rather than the behavioural pattern itself.

However, few studies have looked at specific patterns of non-adherent medication use (as opposed to overall adherence rate) in relation to astimin? outcomes, and it is not clear which ways? Ef cate gorising patterns of medicat or useare most useful

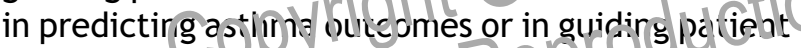
interventiolis.

This study aimed to identify strategic patterns of non-adherent medication use, and to compare asthma outcomes for patients using non-adherent strategies with those for regular medication users.

\section{Methods}

\section{Participants and setting}

A sample of 294 patients, aged 20-60 from the active asthma register of a large semi-rural practice in the UK (Devon) were invited to participate. Asthma care here is delivered by a qualified and experienced asthma nurse working alongside general practitioners (GPs). All patients were at treatment step 2, 3 or 4 of the British Thoracic Society guidelines [1]. Patients using regular oral corticosteroids were excluded, as were pregnant women, patients using other ingested steroid-based medications (not topical creams), and patients with problems affecting their ability to complete questionnaires (e.g. learning difficulties).

\section{Study design}

This was a population-based observational survey, comparing retrospective and cross-sectional asthma outcomes for adherent and non-adherent patient groups.

\section{Measures}

A questionnaire (Box 1 ) was constructed to elicit patterns of medication use. The non-compliance response options were based on preventer medication strategies identified from prior qualitative work [12], other research literature $[9,11,14]$, and discussions of an 'expert panel' comprising three GPs, one asthma nurse, two health psychologists, and one respiratory consultant. The questionnaire was piloted with several patients and amended to improve the acceptability of the wording. The questionnaire followed a decision pathway, exploring first whether ICS was taken regularly every day, then identifying whether any reported nonadherence was unintentional ('forgetting') or intentional, and identifying various patterns of intentional non-adherence.

Prescription recordsprovided a second measure of medicition use. This was simply coded as 'adeGuate (taking 75\% dr more of recommended dosage over the previcus s years) or 'inadequate'.

The main asthma outcome measure was unscheduled service use, assessed from practice records over three years prior to the survey. This was defined as an increase in symptoms resulting in a non-routine visit to the GP or hospital, and leading to either nebulisation, a course of corticosteroid tablets, or increased preventer medication. Events over three weeks apart were counted separately.

The Asthma Quality of Life Questionnaire (AQLQ) [15] was used to assess asthma-specific quality of life over the preceding two weeks.

\section{Procedure}

Eligible patients were posted questionnaires and those not responding within two weeks were sent a reminder. First class stamps, practice letterheads with GP signatures and a return envelope were used to enhance return rates [16]. The study was approved by N\&E Devon Local Research Ethics Committee.

\section{Analysis}

Data were entered into SPSS (Version 11.01). A descriptive analysis of responses was examined by the expert panel to define distinct patterns of 


\section{Box 1: The Patterns of Asthma Medication Use Questionnaire}

These questions are related to the way people take their asthma preventer inhalers.

If you are directed to another question, there will be further instructions there. Different people will finish the questions at different points and there are no right or wrong answers.

How do you actually take your asthma preventer medicine?

1a.I take it regularly, every day, and I never do anything different

(If yes, go to question 2)

b. I sometimes don't take my medicine (If yes, go to question 3)

2. Do you never forget?

If you forget less than once a week, tick here

(You have now finished the questionnaire)

If you forget more than once a week, go to question 3.

3. What is the main reason you don't take your medicine as recommended?

You sometimes forget

(Go to question 4)

You do something else because you think it is better for you

(Go to question 5 )

4. How ofter du you think you fo get (p!case tick one)

About once per week

About twice per week

About every other day

Many days at a time

(You have now finished the questionnaire)

5. Please describe your strategy for taking your preventer medicine

(Tick any that apply)

Take it regularly but less than recommended (e.g. half the dose)

Decide each day whether to use it, depending how I feel

Stop taking your preventer when you feel your asthma is well controlled

(You have now finished the questionnaire)

medication use. Prescription uptake data were used to validate the self-report data, and outcomes were compared between groups with different adherence patterns, and at different levels of severity, as defined by BTS treatment step (which represents the intensity of medication regime prescribed) [1].

\section{Sample size justification}

The study was largely exploratory, and we could not predict the number of groups at the outset. However, based on 3×2 ANOVA comparison of AQLQ scores across three evenly-sized groups and two levels of asthma severity, a sample of 32 per group (total 192) was calculated to have an $80 \%$ chance to detect a significant difference $(p<0.05)$ in the main effect, assuming a clinically meaningful difference of 0.5 points [15] and a standard deviation of 0.9 [15]. Allowing for a $65 \%$ response, we invited 294 patients.

\section{Results}

\section{Responses}

Of those invited, $202(70 \%)$ returned the questionnaires, and $185(64 \%)$ completed the medication strategy questionnaire. The mean age of respondents was 42.1 (SD 10.6, range 21 to 61). 68(34\%) were male. 166 were at BTS treatment step 2, 27 at step 3, and 9 at step 4 .

\section{Patterrs of riledication use}

The ques rolnalie responses were examined by the expert panel; this included making judgements about how (and whether) to code multiple-response combinations (which were given by a small minority of respondents), and making a decision to combine the last two categories in question 5 (see Box 1) into a single category. Consequently, the responses were coded into four distinct, mutually-exclusive response categories (Table 1): patients who take their medication regularly (Regular); those who forgot on two or more occasions per week (Forgetting); those who took medication regularly but at a reduced level (Low-Dosing); and those who varied their strategy in response to symptom levels, in some cases stopping completely (SymptomDirected). Of the 17 who did not complete the

Table 1 Frequencies of reported patterns of medication use.

\begin{tabular}{lcc}
\hline Pattern of Use & Frequency & Percent \\
\hline Regular & 58 & 28.7 \\
Forgetting & 6 & 3.0 \\
Regular Low-Dosing & 26 & 12.9 \\
Symptom-Directed & 92 & 45.6 \\
Not enough data to code & 3 & 1.5 \\
Questionnaire not completed & 17 & 8.4 \\
Total & 202 & 100 \\
\hline
\end{tabular}


Table 2 Cross-tabulation between self-report and prescription data on adherence.

\begin{tabular}{llcr}
\hline & Adherent (self-report) & Non-adherent (self-report) & Total \\
\hline Regular Users (prescription based) N(\%) & $30(52)$ & $22(18)$ & 52 \\
Irregular Users (prescription based) N(\%) & $28(48)$ & $102(82)$ & 130 \\
Total & 58 & 124 & 182 \\
\hline
\end{tabular}

questionnaire, 14 reported having no preventer inhaler (in contrast to the prescription record). To achieve reasonable power in the comparative analyses, the Forgetting group (six patients) was excluded. This left 176 patients with usable data on pattern of medication use.

\section{Validation of self-report questionnaire}

To validate the self-report data, the reported patterns of medication use were classified as adherent (Regular) or non-adherent (Forgetting, LowDosing, Symptom-Directed). Cross-tabulation with prescription data indicated that the majority $(82 \%)$ of those reporting a non-adherent medication strategy were also irregular users according to prescription records (Table 2 ). Of the self-reported adherent patients, $52 \%$ were regular users according to prescription records.

\section{Relationship between pattern of medication} use and asthin outceme

To separate out the impact of asthma severity on outcomes, the sample was split into two groups; those with mild-to-moderate asthma (BTS treatment step 2) and those with moderate-to-severe asthma (BTS steps 3,4). A $3 \times 2$ ANCOVA analysis was then undertaken for each outcome variable, to examine the effects of pattern of medication use (three groups) and asthma severity (two groups). To take into account other possible inter- vening variables, age and gender were entered as covariates.

\section{AQLQ scores}

All 176 patients with usable medication pattern data also completed the AQLQ. Age significantly mediated the AQLQ scores $(F(1,175)=9.08$, $p<0.01$ ), and the results are corrected for this. There were no significant differences between the three patterns of medication use, and pairwise post-hoc comparisons using the Least Significant Differences (LSD) method showed no significant benefit for any pattern. There was a main effect of asthma severity, however, with the moderateto-severe asthma severity group halviing significantly lower AQLQ (lower quality-of life) than the mild-tomoderate roup (Eorrected Mean Diff. 0.99 points; $95 \% 0$. 0.50 to $1.48, p<0.001$ ).

Ther niva: la significant interaction between asthina severity and pattern of medication use $(p=0.24)$, although the numeric trend (Table 3, Fig. 1) suggests that Regular medication users in the moderate-to-severe group had better AQLQ scores than the two non-adherent groups (Corrected Mean Diffs. 0.73 and 0.61 AQLQ points). This is considered worth reporting since: (1) the size of the difference is clinically meaningful [15]; (2) it is consistent with the significant results for unscheduled visits; and (3) the relatively small cell sizes may have not provided sufficient power to detect an interaction effect using this measure.

Table 3 Mean AQLQ scores and number of unscheduled visits.

\begin{tabular}{llrrrr}
\hline Medication Pattern & Treatment Level & $\mathrm{N}$ & $\begin{array}{l}\text { Mean }{ }^{\mathrm{a}}(\mathrm{SD}) \\
\text { AQLQ Score }\end{array}$ & $\mathrm{N}$ & $\begin{array}{l}\text { Mean (SD) } \\
\text { Visits }\end{array}$ \\
\hline \multirow{3}{*}{ Regular } & Mild-to-Moderate & 40 & $5.09(1.33)$ & 38 & $0.63(1.08)$ \\
& Moderate-to-Severe & 18 & $4.60(1.58)$ & 16 & $2.25(2.08)$ \\
& Total & 58 & $4.84(1.42)$ & 54 & $1.11(1.61)$ \\
Symptom-Directed & Mild-to-Moderate & 83 & $5.33(1.04)$ & 78 & $0.73(1.39)$ \\
& Moderate-to-Severe & 9 & $3.99(1.29)$ & 9 & $4.00(2.00)$ \\
& Total & 92 & $4.66(1.13)$ & 87 & $1.07(1.76)$ \\
Low-Dosing & Mild-to-Moderate & 19 & $5.02(1.05)$ & 19 & $0.95(1.81)$ \\
& Moderate-to-Severe & 7 & $3.87(1.44)$ & 7 & $4.71(2.50)$ \\
& Total & 26 & $4.45(1.24)$ & 26 & $1.96(2.60)$ \\
\hline
\end{tabular}

${ }^{\text {a }}$ Corrected for age covariate (evaluated at age $=42.3$ ). 

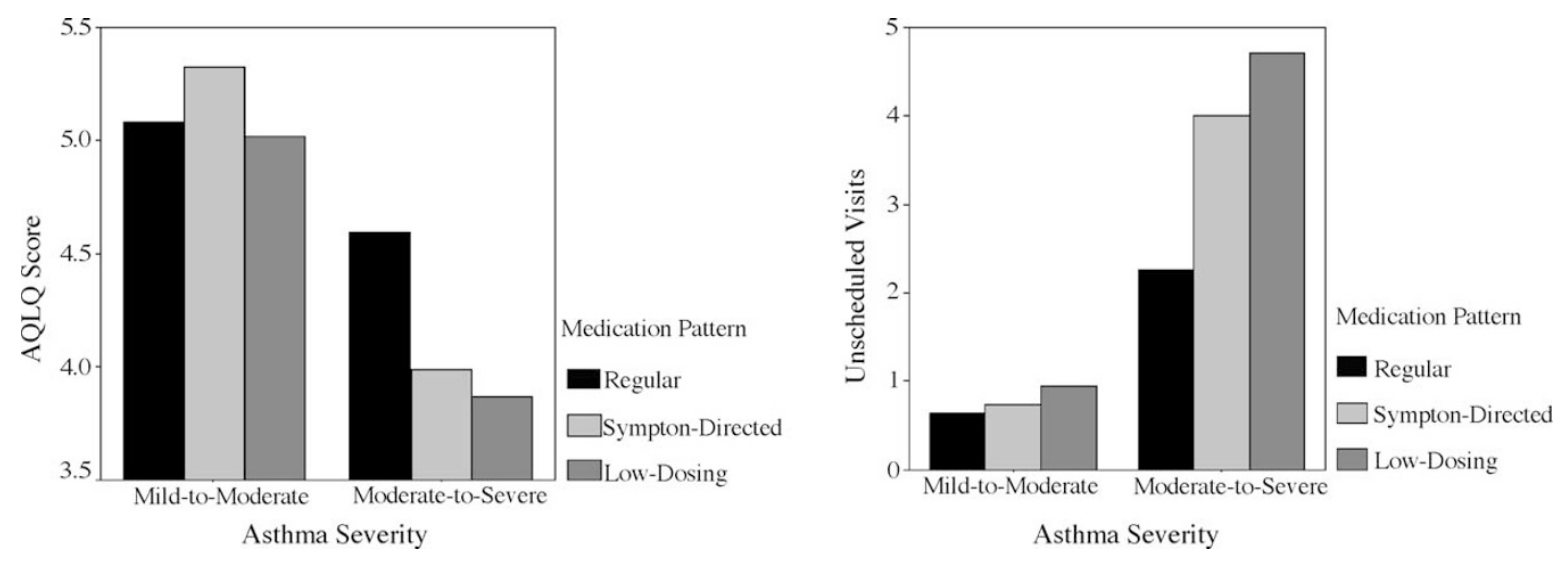

Figure 1 AQLQ scores and unscheduled visits for different medication patterns and asthma severities.

\section{Unscheduled visits}

Of the 176 patients with usable medication pattern data, data on service use was available for 167 . Neither age nor gender significantly mediated the results. There was a significant main effect from pattern of medication use $(F(2,166)=6.76, p<0.005)$, with an increase in unscheduled visits from Regular to Symptom-Directed to Low-Dosing patterns (Table 3, Fig. 1). There was also a main effec from asthma severity, with the moderate to severe group having significa/lallore unscheduled visits

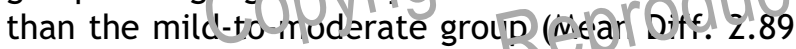
visits; $95 \% \mathrm{Cl}: 2.23$ to $3.54, p<0.001$ ).

There was also a significant interaction between asthma severity and pattern of medication use $(F(1,166)=4.47, p<0.02)$, indicating that whilst there were almost no differences between the different patterns at mild-to-moderate severity, the Regular medication strategy was more successful for more severe asthma (Table 3, Fig. 1).

\section{Discussion}

The questionnaire we developed allowed the identification of three main patterns of medication use: Regular, Low-Dosing, and Symptom-Directed. Most non-adherence seemed to be intentional, reflecting deliberate strategies of either minimising the amount of treatment used regularly (Low-Dosing), or taking medication flexibly depending on recent or current symptoms (Symptom-Directed). Nonintentional non-adherence (Forgetting) was rare.

For mild-to-moderate asthma, non-adherent patterns of medication use seemed to result in acceptable asthma outcomes $(0.73$ unscheduled vis- its per three years, mean AQLQ score 5.2) which were not significantly different from outcomes for adherent medication use. However, for more severe asthma, regular adherence was the most effective strategy, resulting in significantly less unscheduled visits (around two less visits per three-year period) and numerically better asth na qad il wo life scores (improvement of argina $0.6 \mathrm{AQLQ}$ points).

\section{Possitile explanations of the data}

Many patients report that they understand their own asthma better than their GP [17], and adopting a Symptom-Directed or Low-Dosing strategy may in some cases reflect active coping based on in-depth consideration of the patient's individual asthma situation [12]. Given that asthma symptoms vary over time in response to multiple environmental and physiological factors, this type of 'well-reasoned' non-adherence could be appropriate for some patients.

Non-adherent strategies may alternatively be based on a number of other underlying factors, including anxiety about medication (particularly corticosteroid use) [14,18], patient priorities [19], perceived severity of outcomes $[12,18]$, the type and emphasis of advice given by health professionals, and learning from prior experience [12]. In this study, we did not seek to identify the underlying reasons for non-adherence. However, the rationale underlying the medication strategy may influence whether or not the strategy is appropriate (for example, concern about medications may be inappropriate). Understanding the circumstances in which non-adherent strategies are appropriate or inappropriate would therefore be desirable and should be a target for further study. 
Although we attempted to control for underlying asthma severity, treatment level is not a perfect proxy, and so it may be that even within treatment steps, severity explains some of the variation in outcomes. For instance, many of those taking regular medication at step 2 may be those with more severe asthma. Hence, even within treatment steps, regular use may be more important for some patients than others.

Other population-based observational studies using a variety of measures of adherence (including electronic dose-monitoring inhalers) have found no positive association between adherence and good outcomes in adult asthma [5-7,20,21]. This further supports the idea that non-adherence often leads to acceptable outcomes. However, comparative studies indicate that patients with poorly-controlled asthma are more likely to be non-adherent [2-4]. Our data is consistent with these findings also, as the studies comparing outcomes are often focused on hospital inpatients or higher severity patients (where regular medication is the best strategy).

\section{Limitations}

Although we met our sample size requirement, the group sizes were quite uneven. This limited the options for well-powered comparisons betweep the different strategies and for sub-group aralyses. However, the argument that Sy (ii) Eorn- Brected pa-

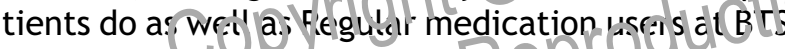
step 2 seemis fairly robust.

Whether or not this sample is representative of the national population may also be questioned. Participants were drawn from one practice in South-West England, which has a predominantly (99.5\%) Caucasian, relatively affluent, semi-rural population.

Although our self-report questionnaire seemed to validate reasonably well against prescription data, there may be doubts about its validity, particularly in identifying Regular users, $48 \%$ of whom did not meet the criterion for regular use. This may reflect a tendency in the prescriptions measure to under-estimate medication used (perhaps due to missing data). However, it is also possible that some patients simply did not want to reveal their nonadherence, and this may potentially affect the results (perhaps diluting the prevalence of positive outcomes for the 'true' regular users).

\section{Implications for practice}

These data do not in any way negate the value of asthma education, or asthma guidelines. However, they suggest that the application of asthma guidelines (which are based largely on non-primary care studies), may involve an over-reliance on ICS for patients with less severe asthma. For mild-tomoderate asthma (BTS step 2) it may be worth accepting symptom-directed medication use and patient-initiated dose-reduction as a viable alternative to regular, as-prescribed medication use. This seems particularly relevant if the patient can provide a clear and reasonable rationale. Instead of targeting non-adherence per se in milder cases, health professionals may therefore be better advised to focus their efforts on patients who have poor asthma outcomes.

It is interesting to note that self-management plans, which are structured around the timely uptitration of ICS medication in response to exacerbations, have proven to be effective [22,23]. An extension of this principle towards the selfmanagement of milder cases would suggest a pattern of up-titration from a medication-free baseline. This would be similar to the Symptom-Directed pattern of medication use observed here, although potentially offering patients a more structured and explicit rationale for this behaviour. It is also worth noting that in practice, some clinicians already agree such patterns (stopning doses when asthma control is good), recognisiing that these work for some nat er ts $1 ? 20$. .

tor thigher treatment steps, regular medicationtaking speph is to to 0. he best strategy.

\section{Future directions}

Further development and validation of the adherence self-report measure against data from electronic dose-recording inhalers would be desirable. The fine detail on which patterns of medication use are appropriate in what circumstances also needs further investigation. The same strategy may suit one patient but not another, depending on whether it is appropriate to the level of severity, the nature of triggers (e.g. seasonal triggers), whether the patient's rationale is appropriate, and the individual dynamics of how the patient's symptoms develop. Once a more detailed understanding is established, further studies could compare different forms of advice-giving in primary care. In particular, the promotion of regular ICS use at step 2 could be compared usefully with a more flexible approach, accepting alternative strategies when these seem appropriate.

\section{Conclusions}

The results suggest that more flexible 'symptomdirected' medication use and patient-initiated 
dose-reduction may be viable alternatives to regular medication use for some step 2 asthma patients.

\section{Acknowledgements}

This project was funded by the Devon Respiratory Research Group using NHS Priorities \& Needs funding from the UK's Department of Health. The authors would like to acknowledge the help of the members of the Devon Respiratory Research Group for commenting on the design and interpretation of the results.

\section{References}

[1] British Thoracic Society/Scottish Intercollegiate Guidelines Network. British Guideline on the Management of Asthma. Thorax 2003;58(Suppl 1):1-94.

[2] Soussan D, Liard R, Zureik M, Touron D, Rogeaux Y, Neukirch F. Treatment compliance, passive smoking, and asthma control: a three year cohort study. Arch Dis Child 2003;88:229-33.

[3] Van Ganse E, Hubloue I, Vincken W, Leufkens HG, Gregoire $J$, Ernst P. Actual use of inhaled corticosteroids and risk of hospitalisation: a case-control study. Eur J Clin Pharm 1997;51:449-54.

[4] Osman LM, Friend JA, Legge JS, Douglas JG. Recue,ts for repeat medication prescriptions ar d fref IEr cy o acute episodes in asthma pationt. As hm 2 1999;36:449-57.

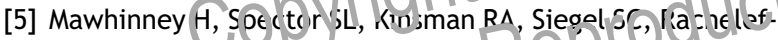
sky GS, Kat $\angle$ RM, et al. Compliar R (1) - lrical trials of two nonbronchodilator, antiasthma medications. Ann Allergy 1991;66:294-9.

[6] Diette GB, Wu AW, Skinner EA, Markson L, Clark RD, MCDonald RC, et al. Treatment patterns among adult patients with asthma: factors associated with overuse of inhaled betaagonists and underuse of inhaled corticosteroids. Arch Int Med 1999;159:2697-704.

[7] Dompeling E, Van Grunsven PM, Van Schayck CP, Folgering $\mathrm{H}$, Molema J, Van Weel C. Treatment with inhaled steroids in asthma and chronic bronchitis: long-term compliance and inhaler technique. Fam Pract 1992;9:161-6.

[8] Rand CS, Wise RA, Nides M, Simmons MS, Bleecker ER, Kusek $\mathrm{JW}$, et al. Metered-dose inhaler adherence in a clinical trial. Am Rev Resp Dis 1992;146:1559-64.

[9] Bender B, Wamboldt FS, O'Connor SL, Rand C, Szefler SJ, Milgrom $\mathrm{H}$, et al. Measurement of children's asthma medication adherence by self report, mother report, canis- ter weight, and Doser CT. Ann Allergy Asthma Immunol 2000;85:416-21.

[10] Rand CS. Adherence to asthma therapy in the preschool child. Allergy 2002;57(s74):48-57.

[11] Osman L, Hyland $M$. Adherence and self management. In: Gibson P, Abramson M, Costabel U, Hensley M, Volmink J, Wood-Baker R, editors. Evidence Based Respiratory Medicine. London: BMJ Books; 2004.

[12] Greaves CJ. Exploring the Asthma Process: Results and Discussion of the Qualitative element of the Adults With Asthma Survey. In: Greaves CJ, editor. Psychosocial Influences On Self-Care And Health Outcomes In Adults With Asthma. Doctoral dissertation. Exeter: University of Exeter; 2002. p. 87-212.

[13] Donovan JL, Blake DR. Patient non-compliance: deviance or reasoned decision-making? Soc Sci Med 1992;34:507-13.

[14] Adams S, Pill R, Jones A. Medication, chronic illness and identity: the perspective of people with asthma. Soc Sci Med 1997;45:189-201.

[15] Juniper EF, Guyatt GH, Ferrie PJ, Griffith LE. Measuring Quality of Life in Asthma. Am Rev Resp Dis 1993;147:832-8.

[16] Edwards P, Roberts I, Clarke M, et al. Increasing response rates to postal questionnaires: systematic review. BMJ 2002;324:1183.

[17] Hyland ME, Finnis S, Irvine SH. A scale for assessing quality of life in adult asthma sufferers. J Psychosom Res 1991;35:99-110.

[18] Horne R, Weinman J. Self-regulation and self-management in asthma: exploring the role of treatment beliefs in exp/aming non-auherence to preventer medicatic . Piy h reali, 2002;17:17-32.

1 s] St even K, Murrison J, Drummond N. Lay versus professional motivation for astorra treatment: a cross sectional, qualitative: lud in a simsle Glasgow general practice. Fam Pract $2002 ; 19(2): 172-7$.

[20] Jones C, Santanello NC, Boccuzzi SJ, Wogen J, Strub $P$, Nelsen LM. Adherence to prescribed treatment for asthma: evidence from pharmacy benefits data. J Asthma 2003;40:93-101.

[21] Wraight JM, Cowan JO, Flannery EM, Town GI, Taylor DR. Adherence to asthma self-management plans with inhaled corticosteroid and oral prednisone: A descriptive analysis. Respirology 2002;7:133-9.

[22] Thoonen BPA, Schermer TRJ, Van Den Boom G, Molema $\mathrm{J}$, Folgering $\mathrm{H}$, Akkermans RP, et al. Self-management of asthma in general practice, asthma control and quality of life: a randomised controlled trial. Thorax 2003;58:306.

[23] Lahdensuo A, Haahtela T, Herrala J, Kava T, Kiviranta K, Kuusisto $P$, et al. Randomised comparison of guided self management and traditional treatment of asthma over one year. BMJ 1996;312:748-52.

[24] Hyland ME. How do patients operate self-management plans? Asthma in General Practice 1997;5:12-4.

\section{Available online at www.sciencedirect.com science@Direct.}

